\title{
Editorial
}

\section{Die Wertschätzung der Tradition und der Respekt vor dem Anderen: Herta Nagl-Docekal zum siebzigsten Geburtstag}

Zum siebzigsten Geburtstag von Herta Nagl-Docekal, den wir am Institut für Axiologische Forschungen gemeinsam mit dem achtzigsten Geburtstag von Enrique Dussel und dem 15-Jahresjubiläum unseres Instituts feiern, habe ich mir lange überlegt, wie ich Herta Nagls philosophische Bestrebungen und ihren Denkstil charakterisieren könnte. Dabei ist mir ein Satz von Olivier Abel eingefallen, der in einem sehr schönen Beitrag die Philosophie von Paul Ricœur als eine "philosophie amicale", eine freundliche und freundschaftliche Philosophie, bezeichnet hat (Abel 2013,1). Und zwar im Sinne einer gutgesonnen Philosophie, die den Dialog und die Vermittlung zwischen verschiedenen, sogar fast inkompatiblen Denkformen sucht. Je mehr ich über all das nachgedacht habe, desto mehr sind mir gewisse thematische Gemeinsamkeiten zwischen Herta Nagl-Docekal und Paul Ricœur aufgefallen. Darauf möchte ich speziell im letzten Teil dieser Würdigung eingehen, die ich in Form einer conversation amicale gestaltet habe.

\section{Aufmerksamkeit und Respekt als Denkstil}

Wenn man das Gesamtwerk von Herta Nagl-Docekal in Betracht zieht, mit der Absicht es unter einer Rubrik zusammen zu fassen, dann wäre man versucht, es als Darstellung und kritische Beleuchtung aktueller Kontroversen in der Philosophie und den Sozialwissenschaften zu bezeichnen. Bei einer aufmerksameren Lektüre entdeckt man jedoch sehr bald, dass dies zu allgemein und unpräzise wäre, denn Nagl-Docekal hat zu all diesen Debatten nicht nur eigene Stellungnahmen bezogen, sondern auch eigene Konzepte und Interpretationen entwickelt. Das Besondere dabei ist, dass sie dies sehr konsequent aus einem ursprünglichen Ausgangspunkt heraus vollzogen hat - dem der Kantischen Philosophie. Ob es sich um Geschichtsphilosophie, philosophische Anthropologie, politische Theorie, Ethik, Ästhetik, feministische Philosophie, Rechtsphilosophie oder Religionsphilosophie handelt, alle ihre Auslegungen und Weiterführungen nehmen implizit oder explizit ihren Ausgangspunkt in der Philosophie Kants. Gewiss, in mancher Hinsicht geht sie auch von Hegel aus, jedoch ist sie primär der kritischen Philosophie Kants verpflichtet und erst danach den Weiterentwicklungen des Denkens der Aufklärung. Herta Nagl-Docekal steht in dieser 
modernen Tradition, befürwortet und verteidigt sie gegen "post-moderne" und andere "antimoderne" Ansätze. Sie tut das jedoch nicht blicklos. Was dabei für sie sehr wichtig ist und was ihren philosophischen Stil insgesamt ausmacht, ist die möglichst adäquate und kontextuelle Wiedergabe der Primärquellen und erst danach deren kritische Beleuchtung aus heutiger Sicht und in Zusammenhang mit kontroversen Debatten. Diese Präzision in der Vorgangsweise äußert sich in ihrer strikten Argumentation, die um logische Schlüssigkeit bemüht ist und die mit einer ausgeprägten Aufmerksamkeit in Verbindung steht. Aufmerksames Lesen, aufmerksames Zuhören, Aufmerksamkeit als Würdigung der Tradition, Aufmerksamkeit als Interesse und Neugier an Neuem, Aufmerksamkeit als Achtung und Respekt vor dem Anderen, bzw. anderen Denkweisen gegenüber - dies ist was nicht nur den gesamten Denkstil, sondern auch die Person von Herta Nagl-Docekal ausmacht. So habe ich sie sowohl bei Vorträgen und Tagungen, als auch in persönlichen Gesprächen erlebt.

Ich bin Herta Nagl-Docekal 1996 am Institut für die Wissenschaften vom Menschen begegnet, als ich dort Visiting Fellow war. In den Diskussionen, die den Dienstagsvorträgen folgten, fiel sie mir sofort auf und zwar gerade mit ihrer extrem klaren und schlüssigen Argumentationsweise. Wenn sie mit der oder dem Vortragenden nicht einverstanden war, dann stellte sie zunächst eine Frage, danach hinterfragte sie die Grundsätze und wies auf gewisse Lücken und Defizite hin. Manche Vortragende kamen bei der Antwort ins Stottern, andere verwickelten sich in zusätzliche Widersprüche, andere wiederum fuhren selbstgefällig fort. Zu diesem Zeitpunkt war ich besonders an einigen Fragen zu feministischer Philosophie interessiert und hatte mir vorgenommen einen Band darüber auf Bulgarisch herauszugeben. Als mich Cornelia Klinger darauf aufmerksam machte, dass Herta Nagl-Docekal die Spezialistin in diesem Bereich sei, ging ich nach einem Vortrag zu ihr und fragte sie, ob wir uns treffen könnten. Sie holte aus einer großen schweren schwarzen Tasche ihren Terminkalender heraus und begann zu blättern. Endlich schien sie einen passenden Termin gefunden zu haben und sagte: "Können Sie am 6. Dezember um $10 \mathrm{Uhr}$ zu mir ins Institut für Philosophie kommen?" Ich schaute sie groß an und dachte: "Meine Güte, nach einem Monat?!" Doch schnell aus meiner Perplexität herausgekommen, antwortete ich: "Aber natürlich. Wo befindet sich denn das Institut?" Sie gab mir ihre Visitenkarte und verabschiedete sich. Als ich nach einem Monat, genau zur verabredeten Zeit, an die Türe ihres Arbeitszimmers klopfte und sie mich freundlich hereinbat, war ich angenehm überrascht, denn ich war mir überhaupt nicht sicher, ob ich sie dort antreffen würde. Ich legte ihr mein Anliegen vor und wir kamen gleich ins Gespräch. Da sie mir aufmerksam zuhörte, schilderte ich ihr meine damaligen Zweifel gegenüber dem Feminismus. Ich sagte ihr, dass ich mich schon vor Jahren mit Beauvoir auseinandergesetzt hatte, auch mit vielen amerikanischen Feministinnen befreundet sei, aber so manche Thesen bedenklich finde. Sie schien 
nicht sonderlich überrascht und erwiderte, dass es da ganz verschiedene Deutungen und Ansätze gäbe und erklärte mir klar und bündig, was sie selbst unter feministischer Philosophie versteht. Wie wir so miteinander sprachen, erinnerte ich mich auf einmal, dass der Leiter des österreichischen Süd-Ost Europa Instituts in Sofia mich gebeten hatte eine Professorin zu einem feministischen Vortrag nach Sofia einzuladen. Ich teilte ihr das mit und es stellte sich heraus, dass er damit genau sie gemeint hatte. Anstatt eines einzelnen Vortrags kamen wir zu dem Schluss gleich eine Konferenz zu veranstalten, die auch tatsächlich 1998 an der Universität Sofia stattfand. Daraus entstand sowohl ein zweisprachiger Sammelband, Die Feministische Philosophie: Perspektiven und Debatten (Raynova/Moser 2000), als auch eine sehr fruchtbare langjährige Kooperation.

Was ich hier besonders hervorheben möchte, bevor ich auf die theoretischen Grundsätze von Herta Nagl-Docekals Werk eingehe, ist ihr Interesse für andere sozio-kulturelle Traditionen und Denkweisen, die sich außerhalb der dominanten westlichen Kultur befinden. Vor zwanzig Jahren, als die Bürgerinnen und Bürger im "anderen Europa" als "arme Verwandte" galten und die "osteuropäischen" Wissenschafterinnen und Wissenschaftler als "Spätzünder" von westlichen Experten, inklusive westlichen Feministinnen, vorwiegend bevormundet und belehrt wurden, hat Herta Nagl-Docekal die Erfahrung der "osteuropäischen" Frauen ernst genommen und ist ihnen mit außerordentlichem Respekt begegnet. Das erkennt man auch aus meinem Interview mit ihr. Auf meine Frage, ob man die Frauenverbände und die Frauenzeitschriften unter dem Sozialismus als "feministisch" bezeichnen könne oder nicht, antwortete sie mir Folgendes:

... ich muss dazu sagen (...) dass ich mich stets dagegen ausgesprochen habe, den Begriff "Feminismus" sehr eng zu definieren, da dies immer dazu führt, dass manche ein Definitionsmonopol in Anspruch nehmen: 'Wir sind die eigentlich feministischen und ihr anderen seid ja im Grunde gar nicht feministisch'. Dabei kommt es auch oft zu einer emotionalisierten Ausgrenzung und Polarisierung, die ich für kontraproduktiv halte. Ich denke also, dass man von Fall zu Fall im Einzelnen untersuchen muss, was die jeweilige Stoßrichtung der Argumentation ist. Die Bezeichnung 'feministisch' ist dann gerechtfertigt, wenn das Interesse auf - ich sage das jetzt sehr allgemein - "Gerechtigkeit für Frauen" gerichtet ist, sei es in der innerfamiliären Aufteilung von Hausarbeit und Erziehung, sei es am Arbeitsplatz oder in der Politik. (...) In diesem Zusammenhang möchte ich noch Folgendes erwähnen: Am Beginn der neunziger Jahre haben wir hier in Wien eine kleine Tagung zum Thema 'Feminismus in West- und Osteuropa' veranstaltet (...) Damals ist mir klar geworden, dass man die im Westen entwickelten Kategorien der feministischen Kritik nicht einfach auf andere Länder übertragen darf. Ich denke, heute eine feministische Debatte in Osteuropa - wenn ich das so pauschal geographisch sagen darf - aufzunehmen, kann nicht in der Form ge- 
schehen, dass Kategorien, die im Blick auf westliche Verhältnisse entwickelt wurden, einfach zur Anwendung gebracht werden. (Raynova 2010, 185-186)

Um zu zeigen, dass man nicht generell sagen könne, dass der Feminismus dieses oder jenes fordere, bringt Nagl-Docekal verschiedene Beispiele und zwar nicht nur im "ostwesteuropäischen" Kontext, sondern auch in Bezug auf Süd-Korea. Feministisches Engagement bedeute in diesem Sinne nichts anderes als "in jedem Land konkret zu untersuchen, ob und wenn ja, auf welche Weise Frauen auf Grund ihres Geschlechts benachteiligt sind" (ebd., 188). Die Notwendigkeit einer kontextuellen und interkulturellen Auslegung wird von ihr nicht nur in Hinblick auf feministische Anliegen artikuliert, sondern auch in $\mathrm{Zu}$ sammenhang mit philosophische Traditionen, wie das z.B. in dem von ihr herausgegebenen Sammelband Postkoloniales Philosophieren: Afrika (Nagl-Docekal/Wimmer 1992) veranschaulicht wird.

Es steht außer Zweifel, dass einer der wichtigsten Beiträge Herta Nagl-Docekals im Bereich der feministischen Philosophie liegt und, dass sie mittlerweile als eine der "Klassikerinnen des modernen Feminismus" (Aguado/ Schmitz 2010) gilt. Da ihre diesbezüglichen Thesen bekannt sind und ich mich zu diesem Thema mehrmals geäußert habe, insbesondere in meinem Buch Feministische Philosophie in europäischem Kontext (Raynova 2010), will ich mich hier nicht wiederholen. Ich möchte vielmehr auf die aktuellen Beiträge NaglDocekals zu einer Neubewertung der Moderne im Lichte des nachmetaphysischen Diskurses eingehen.

\section{Die Grenzen der nachmetaphysischen Moralkonzeptionen}

Die Unterscheidung zwischen Moral, Recht und Religion ist ein Thema, das Herta Nagl-Docekal seit langem beschäftigt und das auch in ihrer kürzlich erschienenen Monographie - Innere Freiheit (2014) - einen zentralen Platz einnimmt. Einige ihrer Hauptthesen wurden bereits in ihrem Artikel "Moral und Religion aus der Optik der heutigen rechtsphilosophischen Debatte" (Nagl-Docekal 2008) vorgestellt. Ihr Ziel liegt darin, zu zeigen, wie mangelhafte Differenzierungen von Recht und Moral zu reduktionistischen Bestimmungen führen können. Als paradigmatisch in diesem Zusammenhang führt sie die Verortung der Moral bei John Rawls an, die von Anfang an zweideutig gewesen sei, da die Fairness, die ja das Grundprinzip der sozialen Kooperation darstelle, zugleich als Moralkonzeption verstanden worden sei. Obwohl Rawls die Moral aus Verfassungsgrundsätzen ableite, scheine er oft auf eine moralische Haltung rekurriert zu haben, die sich außerhalb der Rechtsordnung befinde. Dies sei zum Beispiel der Fall, wenn er fordere, dass die Bürger die Prinzipien von Freiheit und Gleichheit nicht einfach aus Angst vor drohenden Sanktionen 
akzeptieren, sondern "aus moralischen Gründen". Dabei unterstelle er offenbar, "dass Moralität in den Einzelnen bereits vor jeglicher Übereinkunft angelegt ist" (Nagl-Docekal 2008, 844). Wenn er dann versuche die moralische Haltung der Bürger vertragstheoretisch durch "die Tugenden der Kooperation im öffentlichen Leben" zu fundieren, komme er, so Nagl-Docekal, zu einer problematischen Identifikation von Tugend und Moral, bei der er die beiden Begriffe von Tugend, die Kant unterscheide, unberücksichtigt lasse. Kant differenziere nämlich zwischen der Tugend als pragmatischer Orientierung nach Regeln der Klugheit einerseits und der Orientierung am Guten um seiner selbst willen andererseits. Deshalb könne bürgerliches Wohlverhalten auf der Basis einer pragmatischen Ausrichtung zustande kommen ohne jegliche Bezugnahme auf den moralischen Imperativ.

Herta Nagl-Docekal stellt der Auffassung von Rawls Kants Metaphysik der Sitten als eine Alternative gegenüber. Kant gehe davon aus, dass wir zuerst Menschen und erst danach Bürger sind. Als Menschen werden wir im kategorischen Imperativ mit einem Sollen konfrontiert, das alle unsere Handlungen umfasst und auch Verpflichtungen zu Staat und Politik impliziert. Daher müssen wir bei der Vermittlung von Recht und Moral bei der Moral ansetzen, bei der es auch um eine Selbstprüfung geht:

Während das Augenmerk der Bürger auf die soziale Ordnung in ihrer empirisch erfassbaren Dimension (...) gerichtet ist, geht es bei der moralischen Perspektive um die jeweils eigene Motivation. Hier ist zu beachten, dass der kategorische Imperativ als Anweisung zur Selbstprüfung zu verstehen ist (ebd., 848).

In ihrem Buch Innere Freiheit geht Herta Nagl-Docekal jedoch noch weiter. Sie zeigt, dass die Moral nicht nur eine ständige Selbstprüfung impliziert, sondern auch eine "wesentlich umfassendere Pflicht", nämlich, "mitzuwirken an der 'Errichtung und Ausarbeitung einer Gesellschaft nach Tugendgesetzen'", bzw. einem "Tugendstaat" oder "ethischen Staat" (Nagl-Docekal 2014, 162, 168). In ihrer Argumentation greift sie zurück auf Kants Religion innerhalb der Grenzen der bloßen Vernunft, in der er zwischen einem "juridischen" und einem "ethischen" Naturzustand unterscheidet. Kant zufolge nötige das Aufeinanderprallen der Interessen und die eskalierende wechselseitige Bedrohung die Menschen dazu, sich auf das Gehege des Rechts einzulassen und einen bürgerlich-rechtlichen Staat zu errichten, in welchem der juridische Naturzustand überwunden wird (ebd., 163). Da die Moralität auf dieser Ebene jedoch weiterhin bedroht wird, bedarf es, so Kant, eines "vereinigenden Prinzips", d.h. eines Übergangs vom bürgerlich-rechtlichen Staat zu einem "ethischen Staat" oder "Tugendstaat". Nagl-Docekal erläutert dies folgendermaßen: 
So wie die äußere Freiheit erst dann Bestand hat, wenn die Menschen 'gemeinschaftlich unter öffentlichen Rechtsgesetzen leben', so kann auch innere Freiheit nur gemeinsam abgesichert werden (...) Die Überwindung des 'ethischen Naturzustandes' ist also Kant zufolge 'nicht anders erreichbar als durch Errichtung und Ausarbeitung einer Gesellschaft nach Tugendgesetzen'. (ebd., 166)

Die Errichtung einer solchen Gesellschaft könne aber der Einzelne allein nicht vollbringen. Es sei vielmehr die Pflicht der gesamten menschlichen Gattung ihre Bestimmung als solche zu realisieren. Im Gegensatz zu den rechtlichen Gesetzen werde der ethisch-bürgerliche Zustand nicht durch Zwang, sondern durch zwangsfreie Tugendgesetze geregelt. Da es sich dabei nicht um eine äußere, sondern um eine innere, moralisch-verpflichtende Gesetzgebung handle, könne ihr Gesetzgeber nicht aus dem Kreis der Menschen stammen. Kant folgere daraus, dass Gott als Gesetzgeber der Grund aller moralischen Pflichten und Gebote sei und dass der Tugendstaat nur als Kirche gedacht werden könne (ebd., 169). Diese Kirche, erläutert Nagl-Docekal, sei als eine Gemeinschaft wohlgesinnter Menschen zu verstehen, die einander in der Moralität bestärken und das sittliche (göttliche) Gesetz befolgen (ebd., 170-171).

Hier stellt sich für mich die Frage, wie es konkret zur Errichtung eines solchen Tugendstaates kommen kann? Wenn man sich Kants Religionsschrift selbst zuwendet, dann findet man folgende Antwort: Der Mensch ist so fehlbar, dass nur Gott einen Staat nach sittlichen Gesetzen errichten kann. Dennoch sollen die Menschen nicht untätig darauf warten:

Wie kann man aber erwarten, daß aus so krummen Holze etwas völlig Gerades gezimmert werde? Ein moralisches Volk Gottes zu stiften, ist also ein Werk, dessen Ausführung nicht von Menschen, sondern nur von Gott selbst erwartet werden kann. Deswegen ist aber doch dem Menschen nicht erlaubt, in Ansehung dieses Geschäftes untätig zu sein (...) Er muß vielmehr so verfahren, als ob alles auf ihn ankomme, und nur unter dieser Bedingung darf er hoffen, daß höhere Weisheit seiner wohlgemeinten Bemühung die Vollendung werde angedeihen lassen (Kant 1977a, 759).

Desweiteren gibt Kant in der Religionsschrift ein genaues Bild davon, wie der ethische Staat aussehen soll, jedoch keine Indiz dafür, wie aus dem "krummen Holze" etwas, wenn nicht "völlig Gerades", so doch Besseres "gezimmert" werden kann. Hinweise darauf gibt es hingegen in anderen Schriften, wie z.B. in der Kritik der Urteilskraft und in seinen Vorlesungen Über Pädagogik, in denen gezeigt wird, dass die Menschheit durch Erziehung und eine "Kultur der Zucht" zu ihrem moralischen Endzweck gebracht werden kann (Kant 1977b, 389; Kant, 1977c, 706-707). 
So wie das gesamte Verhältnis von Moral und Recht in Kants praktischer Philosophie eines der strittigsten Themen der Kantforschung ist (siehe Pauer-Studer 2000, 127139), so ist für mich das Konzept des "ethischen Staates" in mancher Hinsicht problematisch, insbesondere in Hinblick auf die negativen Folgen, zu denen die verschiedenen historischen Versuche der Realisierung eines Tugendstaates geführt haben. Doch auf dieses umfangreiche Thema kann ich hier nicht weiter eingehen.

Wenn Nagl-Docekal darauf hinweist, dass der äußere Druck keine moralische Besserung bringt, dann stellt sich die Frage wie der ethische Zustand und, demzufolge, die Gemeinde "wohlgesinnter Menschen" aus ihrer Sicht erreicht werden könnte? Sie gibt darauf eine doppelte Antwort. Zum einen betont sie mit Kant die "Leistung der Kultur", die "uns durch die Bearbeitung unserer naturwüchsigen Konstitution an die Moralität heranzuführen" vermag (Nagl-Docekal 2014, 164). Zum anderen versucht sie im Abschlusskapitel über die "religiöse Pluralität im modernen Rechtsstaat" zu zeigen, dass, wenn man den "verborgenen Kern der reinen 'moralischen Religion'", der den verschiedenen Glaubensbekenntnissen und Religionen zugrunde liegt, offen legt (ebd., 197), die Religionen dazu beitragen könnten den Menschen und die Welt zum Besseren zu bewegen.

Im Abschlusskapitel widmet sich Nagl-Docekal der Frage nach der Verbindung zwischen Moral und Religion und stellt die These auf, dass es einer gedanklichen Aneignung der Religion durch die Philosophie bedarf. Den Ausgangspunkt ihrer Argumentation bildet die Habermas'sche These, dass die zunehmende Dominanz des Szientismus, des Naturalismus und des methodischen Atheismus schlussendlich zu einer "entgleisenden Modernisierung" geführt habe, d.h. zu einem wirtschaftlichen Imperialismus ohne globale Perspektiven, zu einer Erschütterung des Normbewusstseins und einer Abbröckelung der Solidarität (ebd., 151ff, 203ff). Durch eine Neulektüre der Religionskonzeptionen von Kant und Hegel stellt Nagl-Docekal als mögliche Bewältigung dieser Krise die Perspektive eines "interfakultären Projekts" in Aussicht, in welchem Philosophie und Theologie sich nicht mehr ausschließen würden.

Es mag auf den ersten Blick seltsam erscheinen, wie zwei so verschiedene philosophische Systeme wie die von Kant und Hegel zusammen gelesen und in eine gemeinsame Denkperspektive gebracht werden können. Diese Vorgangsweise hat für mich jedoch eine erstaunliche Ähnlichkeit mit Ricœurs Auffassung einer notwendigen gemeinsamen Lektüre beider Denker, da wir, seiner Meinung nach, ebenso radikal Nach-Hegelianer wie NachKantianer sind. In diesem Sinne betont er:

Meines Erachtens bestimmen dieser Austausch und diese Permutation noch heute die Struktur der philosophischen Rede. Es ergibt sich daraus die Aufgabe, sie beide [Kant 
und Hegel] immer besser zu denken; man muß sie miteinander denken, den einen gegen den anderen und den einen durch den anderen (Ricoeur 1973, 211).

Ricœurs Methode der Vermittlung ist aber nie bei einem gegeneinander-Lesen stehen geblieben. Es ging ihm immer darum aus Gegenpositionen einen gemeinsamen Kern zu entnehmen und diesen weiter zu entwickeln. Gerade in diese Richtung geht meines Erachtens auch Nagl-Docekal.

Der gemeinsame Kerngedanke von Kant und Hegel, den sie unabhängig von deren unterschiedlichen Prämissen zu enthüllen versucht, ist die Idee der "einen Vernunft" und der Geschichte als Suche nach der Wahrheit und dem Fortschritt im Geiste. Mit anderen Worten, sie zeigt, dass es beiden Philosophen um die Freiheit im Geiste, bzw. um die Verwirklichung der inneren Freiheit geht, aus welcher heraus Moral und Religion allein zu verstehen sind. So betont sie, dass bei Kant das moralische Gesetz von Anfang an in der Vernunft als gesetzgebend angelegt ist. Die Moral sei zwar unabhängig von der Religion, weil der Mensch keine andere Triebfeder des Gesetzes bedarf, um seine eigene Pflicht zu erfüllen, doch gerade durch die Religion erkenne er sich als rückgebunden an einen göttlichen Gesetzgeber (ebd., 157). Nagl-Docekal zeigt, dass eher die Religion der Moral und der Vernunft verpflichtet ist als umgekehrt. Zum einen gelangen die Menschen zu einer gläubigen Haltung aufgrund einer durch das moralische Gesetz bedingten Konfrontation ihrer Endlichkeit, mit dem moralischen Versagen, dem Scheitern und der Sorge, die sie im Alltag erfahren. Zum anderen entsteht die Religion in jedem Menschen aus einem Bedürfnis der Vernunft selbst:

Er [Kant] fasst seinen Gedankengang in der These Zusammen: '(E)s ist moralisch notwendig, dass Dasein Gottes anzunehmen'. Demgemäß begründet die Vernunft in uns 'einen reinen Vernunftglauben', der den Charakter einer unerschütterlichen Überzeugung hat, auf Grund deren die Einzelnen sagen können: '(I)ch beharre darauf und lasse mir diesen Glauben nicht nehmen'. Erst vor diesem Hintergrund wendet Kant sich den vielfältigen im Laufe der Zeit entstandenen Bekenntnissen zu, die er als 'Geschichtsglauben' bezeichnet. Er geht davon aus, dass jedes Bekenntnis in der Überzeugung von der Heiligkeit des Sittengesetzes, d.h. in der reinen moralischen Religion, seine Wurzel hat. (ebd., 196)

Daraus folgert Nagl-Docekal, dass der Vernunftglaube, der in jedem einzelnen Menschen angelegt ist, das Bindeglied zwischen Vernunft, Moral und Religion darstellt.

Obwohl, ihrer Meinung nach, Hegel Kants Moralphilosophie "subjektivistisch unterinterpretiert" und sich vorschnell von ihr abgewendet habe (ebd., 220-221), sieht sie in seiner Deutung der Religion wesentliche Analogien zu Kant. Hegel verstehe die Religion 
als denjenigen Ort, an dem ein Volk für sich bestimme was es für das Wahre halte (ebd. 205). Die Suche nach der Wahrheit und die Erlangung des Bewusstseins, dass die menschliche Natur in der Freiheit des Geistes bestehe, kündigen sich zuerst in der Religion an. Gerade in diesem "Anspruch auf Wahrheit", den man insbesondere bezüglich den Offenbarungswahrheiten antrifft, sieht Nagl-Docekal sowohl eine Verbindung zwischen Wahrheit, Offenbarung und Vernunft, als auch die Analogie zwischen Kant und Hegel. Sie unterstreicht, dass nach Kant eine religiöse Lehre nur dann als geoffenbart gelten kann, wenn sie mit dem von uns als gültig eingesehenen Prinzip der Moral zusammenstimmt, also durch die Vernunft als moralisch untrüglich erfasst wird, und fügt hinzu: "Zu einem analogen Ergebnis gelangt (...) Hegel, der festhält, 'dass die Vernunft der Ort des Geistes sei, wo Gott sich dem Menschen offenbart'" (158). Zu dieser Einsicht sei die christliche Theologie selbst gelangt als mit Luther festgestellt wurde, dass die Glaubenslehre ihren eigentlichen Ort im Denken habe und, dass der Glaube sich im Denken bewähren müsse. Deshalb ist es auch kein Zufall, dass Nagl-Docekal ausdrücklich auf Hegels Äußerung aufmerksam macht: "Wenn die Theologie nicht Philosophie ist, so weiß sie nicht was sie will" (ebd., 219). Die Aktualität von Hegels Religionsphilosophie sieht sie gerade in seinem viel diskutierten Ansatz einer Aufhebung der Religion in der Philosophie und seiner Deutung des Christentums, die "ein differenziertes Instrumentarium bereitstellt: sowohl für eine Analyse der Genese der gegenwärtigen normativen Krise, als auch für die Erwägung eines möglichen Auswegs" (ebd., 204).

Im Gegensatz zum Habermas'schen Vorschlag einer Übersetzung von theologischen Gehalten in eine säkulare Sprache kann die Hegelsche Option, ihrer Meinung nach, als eine Übersetzung in die umgekehrte Richtung verstanden werden. Für Hegel haben Philosophie und Religion denselben Inhalt und deswegen gehe es ihm nicht darum, einzelne christlichtheologische Konzepte neu zu interpretieren, sondern darum, die gesamte christliche Lehre auf den Begriff zu bringen und die christliche Auffassung von Geist und Trinität denkend einzuholen (ebd., 217-218). Die Philosophie könne zwar die Religion nicht ersetzen, denn sie vermag keine Gemeinde zu bilden, aber sie könne den Glaubensgemeinschaften "einsichtig machen, welche Relevanz die in ihren Lehren angelegten subtilen Erwägungen für gegenwärtige Problemlagen haben (...) Für die Theologie kommt es dabei darauf an, Philosophie auf dem größten Differenzierungsniveau der Gegenwart zu rezipieren" (ebd., 219). Auf diese Weise würden sich, so Nagl-Docekal, Philosophie und Theologie nicht mehr gegenüber stehen, sondern könnten gemeinsam in einem interdisziplinär ausgerichteten "interfakultären Projekt" an einem unverkürzten, antireduktionistischen Begriff von Humanität arbeiten. 


\section{Die Tradition und ihre Rezeptionsweisen}

Es ist hier weder meine Absicht, noch meine Aufgabe zu diskutieren, ob man die Religion, bzw. die Theologie in die Philosophie "aufheben" kann oder soll, selbst wenn es nur um ihren kognitiven Anteil geht, und ob man die Religion nur dann richtig verstehen und die religiösen Antagonismen zu überwinden vermag, wenn man den "verborgenen Kern der reinen 'moralischen Religion'" aufdeckt (ebd., 197). Stattdessen möchte ich zum einen auf den Beitrag von Herta Nagl-Docekal in Bezug auf die Reaktualisierung der Kantischen und Hegelschen Ansätze hinweisen, zum anderen einen Vergleich mit Paul Ricœurs Kant-Interpretation ziehen, die eine alternative Verbindung zwischen Moral und Religion anbietet.

Der wesentlichen Beitrag Nagl-Docekals besteht, meines Erachtens, in der Thematisierung des Bedarfs einer gedanklichen Einholung der Religion. Ich finde diesen Ansatz, insofern er nicht als ein "entweder (Hegel) - oder (Habermas)" gelesen wird, besonders hilfreich in methodologischer Hinsicht. Durch die Aufarbeitung der Hegelschen religionsphilosophischen Perspektive wird nämlich klar, dass der hermeneutische Vorgang schrittweise zu erfolgen hat, d.h. dass es zuerst einer philosophischen Übersetzung der religiösen Narrative und Lehren bedarf bevor eine Übersetzung in säkulare Sprache unternommen werden kann.

An dieser Stelle möchte ich darauf hinweisen, dass Paul Ricœur einen Weg eingeschlagen hat, der gewisse Gemeinsamkeiten, aber auch wesentliche Unterschiede zu NaglDocekals Versuch einer Reaktualisierung der Moderne aufweist. Ich werde hier der Klarheit halber nur auf drei Punkte seiner Kant-Interpretation eingehen, die ich in direkten Bezug zu Nagl-Docekals Interpretation der Relation zwischen Moral und Religion, bzw. Philosophie und Religion, stellen werde.

Es ist bekannt, dass der frühe Ricœur die ethischen und religiösen Dimensionen der menschlichen Freiheit in Zusammenhang mit der Kantischen Frage nach dem radikal Bösen artikuliert hat (Ricœur 1971a, 104-109, 183ff). Was aber in der Ricœurforschung außer Acht gelassen wird, ist seine Konzeption der Notwendigkeit einer "zweiten kopernikanischen Revolution". Wenn, so Ricœur, bei der Kantischen kopernikanischen Revolution der Bezug "Freiheit - Natur" in den Vordergrund trete, so sei noch eine "zweite" kopernikanische Revolution vonnöten, die den Bezug "Freiheit - Transzendenz" ins Zentrum stelle. Anders gesagt, bei der Kantischen kopernikanischen Revolution sei die objektive Welt auf das Cogito zentriert: Das Objekt ist ein solches nur für ein Subjekt. In der "zweiten kopernikanischen Revolution", die er vorschlägt, um zu einem tieferen Verständnis der Subjektivität zu gelangen, solle das Zentrum zur göttlichen Transzendenz hin verschoben werden, 
sodass die Subjektivität als Teil eines allumfassenden Ganzen wahrgenommen werde. Erst durch diesen Bezug zwischen Subjektivität und göttlicher Transzendenz würden wir einen Zugang zum "ontologischen Mysterium" bekommen, jedoch nicht mittels der Vernunft, denn das Mysterium entziehe sich jeder rationalen Erkenntnis, sondern durch eine Art poetischer Bewunderung, in der sich die Welt durch die Chiffren der Transzendenz (Jaspers) offenbare. Diese "Poetik der Freiheit", die Ricœur in der lyrischen Dimension der Seele ansetzt, steht für ihn als Zugang zur Transzendenz höher als die Moral, die sich auf der sachlichen Ebene der Normativität, der Bejahung des Guten, befindet (Ricœur 1949, 449). Aus dieser Perspektive heraus zeigt Ricœur in der Symbolik des Bösen, dass wir der Moderne zwar das klare Denken verdanken - das der formalen Logik, der Exegetik und der Phänomenologie der Religion -, dass aber die Moderne zugleich einen Verlust darstelle: "Vergessenheit der Hierophanien, Vergessenheit der Zeichen des Heiligen, Verlust des Menschen selbst, insoweit er dem Heiligen angehört" (Ricœur 1971b, 397).

Kurz gesagt, im Unterschied zu Kant und Nagl-Docekal werden beim frühen Ricœur Moral und Religion nicht aus der Vernunft heraus interpretiert, sondern aus dem Mysterium und der Transzendenz. Der Gedanke, den Ricœur gemeinsam mit Kant und mit NaglDocekal teilt, besteht in der Notwendigkeit einer gedanklichen Einholung religiöser Inhalte durch die Philosophie als Hermeneutik oder hermeneutica sacra (siehe Nagl-Docekal 2014, 158).

Das Projekt einer Poetik der Freiheit, das ähnlich wie Jaspers eine Begründung des "philosophischen Glaubens" anstrebt, wird später von Ricœur aus verschiedenen Gründen aufgegeben. Einer davon ist meines Erachtens die Unmöglichkeit einer philosophischen Einholung der Chiffren der Transzendenz, da die Poetik des Glaubens in Bereiche eindringt, die sich außerhalb der Kompetenzen der Philosophie befinden. Bei seiner Abwendung von der christlichen Philosophie stützt sich Ricœur in seinem Spätwerk auf Kant, der die ontologische und kosmologische Unmöglichkeit eines Gottesbeweises nachgewiesen habe (Ricœur 1994, 19). Dies erklärt zum Teil auch seine vehemente Ablehnung jeglicher religiös gefärbter Philosophie (Ricœur/ Raynova 2004, 101-106). So endet Das Selbst als ein Anderer mit dem Grundsatz, dass Gott eine Aporie des Anderen darstelle, bei welcher der philosophische Diskurs stehen bleiben müsse (Ricœur 1996, 426). Man könnte nun annehmen, dass Ricœurs Ausklammerung Gottes aus dem philosophischen Diskurs zu einer Rückbindung an die Vernunft und zu einer Inthronisierung der Moral führen würde. Doch genau das passiert nicht. Die "kleine Ethik", die im Zentrum des Spätwerks Das Selbst als ein Anderer steht, ist eine kritische Auseinandersetzung mit Kant. Ricœur unterscheidet zwischen der Ethik als Zielsetzung unserer Handlungen (Teleologie) und der Moral als deren normativem Gerüst (Deontologie). Die praktische Weisheit als Endzweck der Ethik 
ziele darauf ab, "die phronèsis im Sinne des Aristoteles über die Moralität im Sinne Kants mit der Sittlichkeit im Sinne Hegels zu versöhnen" (ebd., 351). Deshalb versteht er die Ethik als "Ausrichtung auf das 'gute Leben' mit Anderen und für sie in gerechten Institutionen" (ebd., 210). Ricœurs Absicht ist es, das Primat der Ethik vor dem der Moral als Sittengesetz nachzuweisen und somit $\mathrm{zu}$ einer Balance zwischen Formalismus und Kontextualismus zu kommen. Die Moral mit ihren Pflichten und Normen habe zwar eine wichtige Korrekturfunktion, um egoistische Übergriffe zu unterbinden, aber sie sei nicht das Alpha und Omega unserer sittlichen Praxis. Sie sei auch nicht an einen göttlichen Gesetzgeber gebunden, sondern an die phronèsis als kritische Instanz, die uns ein "moralisches Situationsurteil" ermögliche (ebd., 351).

Ricœur erläutert an diversen Stellen, dass der Schnittpunkt und der gemeinsame Kern zwischen Philosophie und Religion/Theologie in der Ethik besteht (siehe Raynova 1999, 78-80). Daraus könnte man folgern, dass die Religionsphilosophie von der Ethik her zu verstehen ist. In einem wenig bekannten Aufsatz - "Une herméneutique philosophique de la religion: Kant" (1992) -, der speziell Kants Religionsschrift gewidmet ist, zeigt Ricœur jedoch, dass die Philosophie der Religion weder aus der theoretischen, noch aus der praktischen Philosophie, sprich aus der Ethik, ableitbar ist. Schon im Titel bezeichnet er Kants Religionsschrift als eine "philosophische Hermeneutik der Religion", um klar zu stellen, dass sie keine Weiterführung der Kantischen Kritik ist, da es hier nicht um die Frage nach Gott gehe, sondern um die nach der Religion (Ricœur 1994, 19). Diese Kantinterpretation ist von besonderer Bedeutung, weil hier der hermeneutische Ansatz von Kants Religionsphilosophie betont wird, wie dies auch Nagl-Docekal macht. Darüber hinaus artikuliert Ricœur jedoch die grundlegende Bedeutung der Religion für die Philosophie.

Von Ricœurs vielschichtiger Analyse möchte ich hier zwei Punkte hervorheben, die im Lichte von Nagl-Docekals Kantinterpretation von Interesse sind. Nämlich, die Rolle der kirchlichen Institution in Hinsicht auf die Etablierung eines ethischen Staates und die Bedeutung der Religion als nichtphilosophische Quelle und Ressource der Philosophie.

Ricœur betont, dass bei Kant der Glaube in seiner geistlichen Dimension untrennbar mit der institutionellen Form der Religion verbunden sei, sodass die Ankündigung des Reichs Gottes und dessen Einrichtung auf Erden als Kirche schon in der Struktur des Glaubens inbegriffen sei. Doch erst mit der Frage nach dem Kult und dem Priesteramt trete der institutionelle Aspekt der Religion ins Zentrum. Auf dieser Ebene erreiche der Kampf des guten und des bösen Prinzips bei Kant seinen Höhepunkt in Form eines Gegensatzes zwischen dem "wahren" und dem "falschen" Glauben, folglich zwischen der "wahren" Kirche und ihren historischen Verunstaltungen. Ricœur erläutert, dass die Institution dem Glauben das hinzufüge, was Hannah Arendt "eine öffentliche Erscheinung" nenne und dass dies von 
zentraler Bedeutung sei, weil das gute Prinzip bei Kant nur in der Inkarnation eines öffentlichen, klerikalen Körpers Souveränität erlangen könne. Ricœur stellt sich hier die Frage, ob die Kirche tatsächlich diejenige Gemeinschaft ist, welche die Souveränität des guten Prinzips garantieren könne. Kant jedenfalls komme zu dieser Schlussfolgerung dadurch, dass er seine rationalistische Interpretation der christlichen Dogmen auf der Voraussetzung einer Affinität zwischen einem zeitlosen, göttlichen Prinzip und einer historischen Realität aufgebaut habe, die sich für ihn am deutlichsten in der Moderne offenbare. Kants Aufgabe sei also gewesen, Hinweise oder Zeichen für diese Affinität zu finden (ebd., 38). Er hätte also nicht nach einer "unsichtbaren" Kirche gesucht, sondern nach Zeichen einer authentischen Gemeinschaft von sichtbarer Kirche, sprich nach Anzeichen eines ethischen Staates.

Ricœur beendet seine Ausführungen damit, dass die Philosophie auf die Religionsthematik angewiesen sei, weil sich die Wurzel des Bösen außerhalb der Vernunft befinde und die Frage nach der Hoffnung nur im Rahmen einer Religionshermeneutik beantwortet werden könne (ebd., 40).

Die grundlegende methodologische Konsequenz von Paul Ricœurs und Herta NaglDocekals Kantinterpretationen besteht meines Erachtens darin, klare Grenzen zwischen Recht und Moral, Religion und Moral, Philosophie und Theologie zu ziehen, jedoch auch deren Schnittstellen interpretativ aufzuzeigen. Obwohl für beide die Reaktualisierung der Bedeutung der Moderne durch Rückgriff auf Kant und Hegel ein zentrales Anliegen ist, sind sie in vieler Hinsicht zu verschiedenen Ergebnissen gelangt. Dies zeigt zum einen, dass, auch wenn man sich in derselben Tradition bewegt und an der Leistung der Vernunft festhält, ein "Konflikt der Interpretationen" nie ausgeschlossen werden kann, zum anderen aber, dass Philosophie nicht unabhängig von der jeweiligen Kultur und Tradition, ja der eigenen Geschichte und Erfahrung betrieben werden kann, so abstrakt sie auch sein mag. Ob man nun in der Tradition bleibt und sich bemüht diese zu erneuern und zu erweitern, ob man Wege sucht, sie mehr oder weniger radikal zu überschreiten oder, ob man sie mit anderen Denkweisen in Verbindung setzt und dann dazwischen vermittelt - das alles sind Fragen der Methode und des Denkstils. Herta Nagl-Docekals Denkansatz verbindet alle diese philosophischen Vorgangsweisen auf die eine oder die andere Art. Sie zeigt dabei, dass man eine Tradition nicht überschreiten kann ohne sich damit ernsthaft auseinander zu setzen und, dass die "neuen" Lösungsansätze oft in angeblich überwundene Denkmodelle hinein kippen. Etwas Ähnliches konstatiert auch Derrida in L'écriture et la différence wenn er darauf hinweist, dass die Kritik der Metaphysik einen Zirkel enthält, da sie selbst mit metaphysischen Begriffen operiert (Derrida 1967, 413). Das wovor Nagl-Docekal noch zusätzlich warnt, ist die Art und Weise wie die große Tradition der Moderne rezipiert wird, nämlich das Ausarten in eine Polarisierung zwischen "verkrusteten Einschätzungen" und 
"abgeschotteten professionalisierten Diskursen" (Nagl-Docekal 2014,11). Leider trifft diese Diagnose nicht nur auf die Rezeption moderner Denker zu. Ist sie nicht vielmehr eine zunehmende Krankheit der Gegenwartsphilosophie selbst?

Yvanka B. Raynova (Wien / Sofia)

\section{Literaturangaben}

Abel, Olivier. "Paul Ricœur et l'amitié", 2013, online: http://olivierabel.fr/ricoeur/paul-ricoeur-et-1amitie.php

Aguado, Maria I. Pena / Bettina Schmitz (Hg.). Klassikerinnen des modernen Feminismus. Aachen: ein-FACH Verlag, 2010.

Derrida, Jacques. L'écriture et la différence. Paris: Seuil, 1967.

Kant, Immanuel. Die Religion innerhalb der Grenzen der bloßen Vernunft (Werke in zwölf Bänden, Band 8). Frankfurt am Main: Suhrkamp, 1977a.

Kant, Immanuel. Kritik der Urteilskraft (Werke in zwölf Bänden, Band 10). Frankfurt a.M.: Suhrkamp, 1977b.

Kant, Immanuel. Über Pädagogik (Werke in zwölf Bänden, Band 12). Frankfurt a.M.: Suhrkamp, 1977c.

Nagl-Docekal, Herta/ Franz Wimmer. Postkoloniales Philosophieren: Afrika. Wien: Oldenbourg, 1992.

Nagl-Docekal, Herta. "Moral und Religion aus der Optik der heutigen rechtsphilosophischen Debatte". - Deutsche Zeitschrift für Philosophie, 56 (2008) 6, 843-855.

Nagl-Docekal, Herta. Innere Freiheit. Grenzen der nachmetaphysischen Moralkonzeptionen. Berlin: De Gruyter, 2014.

Pauer-Studer, Herlinde. Autonom leben. Reflexionen über Freiheit und Gleichheit. Frankfurt am Main: Suhrkamp, 2000.

Raynova, Yvanka B. "Entre la régression et l'eschatologie: Philosophie et théologie dans la phénoménologie herméneutique de Paul Ricœur." In: Charlotte Methuen (Ed.). Time - Utopia Eschatology. Peeters: Leuven, 1999, 65-80.

Raynova, Yvanka B. / Susanne Moser u.a. Die Feministische Philosophie: Perspektiven und Debatten. Sofia: Nauka i izkustvo, 2000.

Raynova, Yvanka B. Feministische Philosophie in europäischem Kontext. Wien u.a.: Böhlau, 2010. 
Ricœur, Paul. Le volontaire et l'involontaire. Paris: Aubier, 1949.

Ricœur, Paul. Die Fehlbarkeit desMenschen. Phänomenologie der Schuld I. Freiburg/München: Karl Alber Verlag, 1971a.

Ricœur, Paul. Die Symbolik des Bösen. Phänomenologie der Schuld II. Freiburg/München: Karl Alber Verlag, 1971b.

Ricœur, Paul. "Die Freiheit im Licht der Hoffnung". In: Ders. Hermeneutik und Strukturalismus. Der Konflikt der Interpretationen I. München: Kösel Verlag, 1973, 199-226.

Ricœur, Paul. "Une herméneutique philosophique de la religion: Kant", in: Ders. Lectures 3. Aux frontières de la philosophie. Paris: Seuil, 1994, 19-40.

Ricœur, Paul. Das Selbst als ein Anderer. München: Wilhelm Fink Verlag, 1996.

Ricœur, Paul/ Yvanka B. Raynova. "Der Philosoph und sein Glaube".- Deutsche Zeitschrift für Philosophie 52 Jg. (2004) Heft 1, 85-112. 